\title{
Cornu cutaneum of Pinna
}

\author{
Satish Kumar C, ${ }^{1}$ Bharathi K $V^{2}$ Kumaran Ramesh Colbert, ${ }^{1}$ Sophia A1 ${ }^{1}$
}

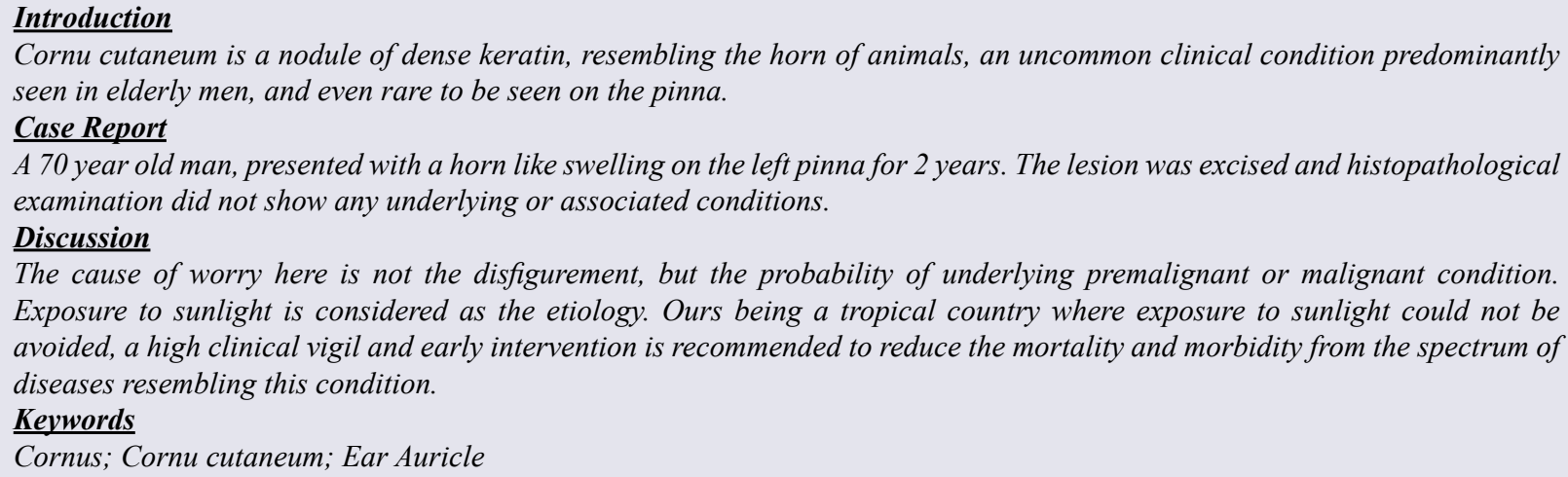

$\mathrm{C}$ ornu cutaneum used interchangeably with sebaceous horn or cutaneous horn is a projectile, conical, nodule of dense keratin, resembling the horn of animals. ${ }^{1}$ This is an uncommon clinical condition predominantly seen over the sun exposed areas of face and scalp among men in 6th or 7th decade of life. ${ }^{2}$ Although it appears as benign lesion, a prompt diagnosis by excision and histopathological examination is advocated to rule out underlying premalignant or malignant conditions. ${ }^{3}$

\section{Case Report}

A 70 year old male presented to the ENT opd with complaints of a horn like swelling on the left ear for 2 years, started as a small painless nodule over the pinna, became conical and progressively enlarged to the present size. There was no history of trauma, bleeding, itching, discharge, ulceration, discoloration, satellite lesions or sudden increase in the size of lesion.

On examination a horn shaped swelling of size $2 \times 1 \times 0.5 \mathrm{~cm}$, dark brown in colour, was seen arising from the rim of helix left pinna at the junction of upper and lower halves. No ulcerations, scars, discharge, satellite lesions or nodules were seen around the swelling. (Fig. 1)
Swelling was non-tender, not warm, hard in consistency, non compressible, non-pulsatile and fixed to the skin of pinna but free from the underlying cartilage.

The lesion was excised under local anesthesia with a rim of normal skin at the base. The defect was closed primarily with 2.0 monofilament nylon. Patient was discharged with a course of oral antibiotics on the same day and followed up every 6 weeks for 8 months and every 6 months thereafter. The follow up was uneventful with no local recurrence.

On histopathological examination, the sections showed skin with marked hperkeratosis, the epidermis was hyperplastic with papillomatosis. Dermoedpidermal junction showed dense infiltration of mixed inflammatory infiltrates composing of lymphocytes and eosinophils. (Fig. 2, 3)

1 - Department of ENT, Indira Gandhi Medical College and Research Institute, Puducherry

2 - Department of Pathology, Indira Gandhi Medical College and Research Institute, Puducherry

Corresponding author:

Dr Satish Kumar C

email: docsatkumar@gmail.com 


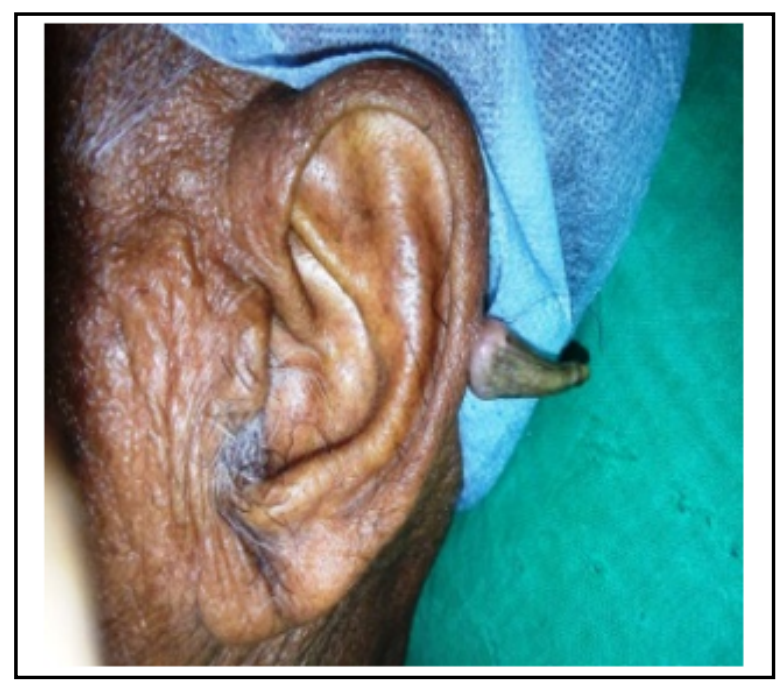

Fig.1: A horn shaped swelling arising from the helix of left pinna

\section{Discussion}

Cornu cutaneum is a descriptive clinical and histological term for horn like hyperkeratotic lesion, used interchangeably with sebaceous horn or cutaneous horn. Cornu cutaneum is characterized by projectile, conical, nodule, composed of dense keratin, resembling the horn of animals. ${ }^{1}$ Histological differentiation is made by the presence of superficial hyperkeratotic epidermis, dermis, and centrally positioned bone in animal horns. ${ }^{4}$

Elderly men in 6th or 7th decade are commonly affected over the sun exposed areas. ${ }^{2}$ Mrs. Margaret Gryffith, an elderly welsh woman was the first ever documented case of cornu cutaneum from London in 1588. However a London based surgeon, Everard Home in 1791 was the earliest to describe the observations on cutaneous horn. ${ }^{5}$ A well documented case report with adequate histology was first described by Farris from Italy. ${ }^{6}$

The cause of worry in this condition is not the horn but the spectrum of associated conditions including - actinic keratosis, viral warts, pyogenic granuloma, keratoacanthoma, seborrheic keratosis, verruca vulgaris, discoid lupus erythermatosus, basal cell carcinoma, and squamous cell carcinoma. ${ }^{7,8}$ In the study by $\mathrm{Yu}$ et al comprising 643 subjects, $39 \%$ of cutaneous horns had associated premalignant or malignant lesion and $61 \%$

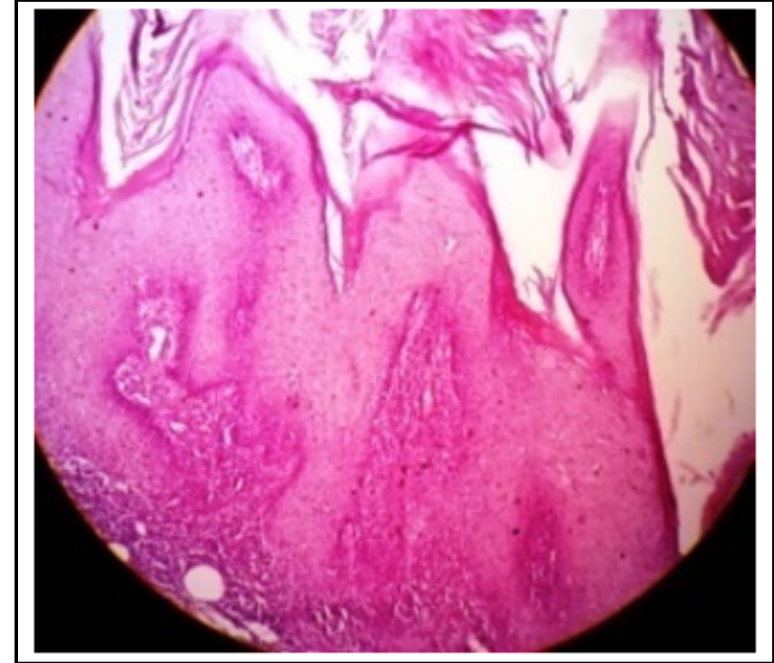

Fig. 2. Skin showing epidermal hyperkeratosis with papillamatosis and hyperkeratosis $(\mathrm{H} \& \mathrm{E} \times 40)$

had benign lesions. ${ }^{3}$ Such observation were made in two other studies, wherein $23-37 \%$ cases had actinic keratosis or Bowen's disease (premalignant) and $16-20 \%$ cases had an underlying malignant etiology. ${ }^{5,9}$ However in our patient no associated lesions were documented.

Exposure to sunlight is considered as the etiology for cutaneous horns as is the case with other skin lesions. ${ }^{10}$ Surgical excision with a rim of surrounding normal tissue is the treatment of choice. The wound is closed

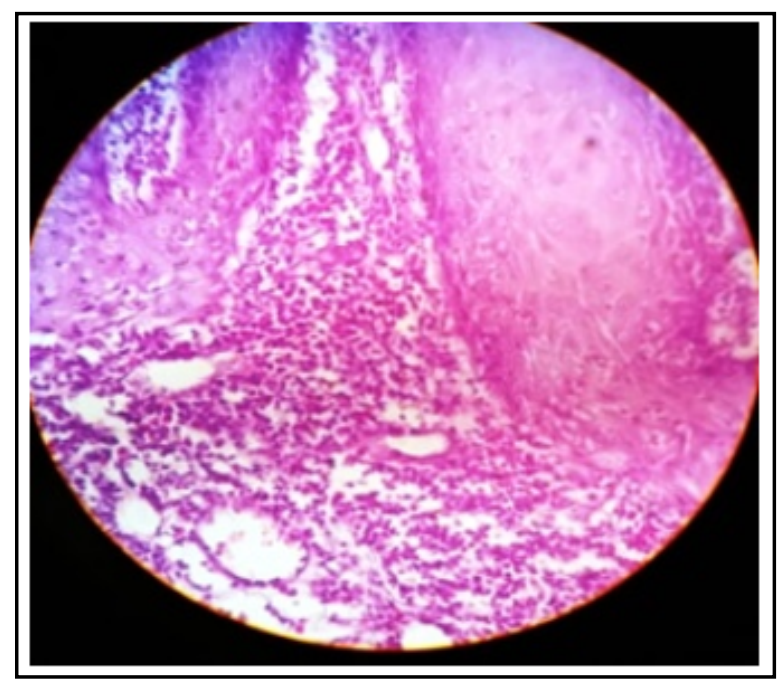

Fig. 3: Dense mixed acute and chronic inflammatory cell infiltrate in the papillary dermis, extending up to the dermo-epidermal margin (H\&E x100) 
primarily or using a flap depending on the size of defect. ${ }^{3}$

Ours being a tropical country where exposure to sunlight could not be avoided, and the high incidence of cutaneous horns (potentially malignant) in elderly patients who already have risk of other malignancies, we recommend high clinical vigil and early intervention to reduce the mortality and morbidity associated with the spectrum of diseases resembling this condition.

\section{References}

1. Korkut T, Tan NB, Oztan Y. Giant cutaneous horn: A patient report. Ann Plast Surg. 1997; 39:654-5

2. Souza LN, Martins CR, de Paula AM. Cutaneous horn occurring on the lip of a child. Int J Paediatr Dent. 2003; 13:365-7

3. Yu RC, Pryce DW, Macfarlane AW, Stewart TW. A histopathological study of 643 cutaneous horns. Br J Dermatol. 1991; 124:449-52

4. Michal M, Bisceglia M, Di Mattia A, Requena L, FanburgSmith JC, Mukensnabl P, et al. Gigantic cutaneous horns of the scalp: lesions with a gross similarity to the horns of animals: a report of four cases. Am J Surg Pathol. 2002; 26:789-94

5. Home BJE, Hunter J. cutaneous horns: a historical review. Am J Dermatopathol. 2001; 23:362-9

6. Farris G. Histological considerations on a case of a voluminous cutaneous horn. Minerva Dermatol. 1953; 28:159-65

7. Akan M, Yildirim S, Avci G, Akoz T. Xeroderma pigmentosum with a giant cutaneous horn. Ann Plast Surg. 2001;46:665-6

8. Farris G. Histological considerations on a case of a voluminous cutaneous horn. Minerva Dermatol. 1953; 28:159-65

9. Schosser RH, Hodge SJ, Gaba CR, Owen LG. Cutaneous horns: a histopathologic study. South Med J. 1979; 72:1129-31

10. Bart RS, Andrade R, Kopf AW. Cutaneous horns. A clinical and histopathologic study. Acta Derm Venereol. 1968; 48:507-15. 